\title{
Status and Prospects of Heavy Element Nuclear Chemistry Research at JAERI
}

\author{
Y. Nagame, ${ }^{*}$ M. Asai, H. Haba, K. Tsukada, S. Goto, ${ }^{\dagger}$ M. Sakama,${ }^{\ddagger}$ I. Nishinaka, A. Toyoshima, \\ K. Akiyama, and S. Ichikawa \\ Advanced Science Research Center, Japan Atomic Energy Research Institute, Tokai, Ibaraki 319-1195, \\ Japan
}

Received: March 3, 2002; In Final Form: May 9, 2002

The present status of heavy element nuclear chemistry research at JAERI (Japan Atomic Energy Research Institute) is reviewed. Successful production of the transactinide nuclei, ${ }^{261} \mathrm{Rf}$ and ${ }^{262} \mathrm{Db}$ via the reactions of ${ }^{248} \mathrm{Cm}\left({ }^{18} \mathrm{O}, 5 \mathrm{n}\right)$ and ${ }^{248} \mathrm{Cm}\left({ }^{19} \mathrm{~F}, 5 n\right)$, respectively at the JAERI tandem accelerator is presented. Aqueous chemistry of Rf is being carried out with a newly developed rapid ion-exchange separation apparatus. Anion-exchange behavior of $\mathrm{Rf}$ in $\mathrm{HCl}$ solution is shortly discussed. Recent experimental results on decay studies of neutron-deficient actinide nuclei using the gas-jet coupled JAERI-ISOL are also given. Prospects for the near future studies are briefly considered.

\section{Introduction}

Nuclear chemistry study of the transactinide elements in Japan is currently being performed using the JAERI tandem accelerator. We have developed some experimental apparatuses: a beam-line safety system for the usage of the gas-jet coupled radioactive ${ }^{248} \mathrm{Cm}$ target and recoil chamber, a rotating wheel catcher apparatus for the measurement of $\alpha$ and spontaneous fission decay of transactinides, and an automated rapid ionexchange separation apparatus based on the high performance liquid chromatography coupled with an on-line $\alpha$-particle detection system. ${ }^{1}$

The schematic of the experimental setup for the study of the transactinide elements is shown in Figure 1. The transactinide nuclei, 78-s ${ }^{261} \mathrm{Rf}$ and $34-\mathrm{s}{ }^{262} \mathrm{Db}$ which are commonly used for recent chemical studies of element 104 and 105, have been successfully produced for the first time in Japan through the reactions of ${ }^{248} \mathrm{Cm}\left({ }^{18} \mathrm{O}, 5 n\right)$ and ${ }^{248} \mathrm{Cm}\left({ }^{19} \mathrm{~F}, 5 n\right)$, respectively. The production cross sections for each reaction have been accurately measured with the newly developed rotating wheel catcher apparatus MANON (Measurement system for Alpha particle and spontaneous fissioN events $\mathbf{O N}$ line). ${ }^{2}$

On-line anion-exchange experiments of $\mathrm{Rf}$ together with the group 4 elements $\mathrm{Zr}$ and $\mathrm{Hf}$ in $\mathrm{HNO}_{3}$ and $\mathrm{HCl}$ solutions have been conducted with AIDA (Automated Ion exchange separation apparatus coupled with the Detection system for Alpha spectroscopy). The results have clearly shown that ion-exchange behavior of $\mathrm{Rf}$ is quite similar to that of $\mathrm{Zr}$ and $\mathrm{Hf}$, and is different from that of the tetravalent pseudo-homologue Th, indicating $\mathrm{Rf}$ is the member of the group 4 elements. ${ }^{3}$

Studies of $\alpha / \mathrm{EC}$ (electron capture)-decay properties of neutron-deficient actinide nuclei have been carried out using the gas-jet coupled JAERI-ISOL system and recently the new isotopes ${ }^{233,236} \mathrm{Am}$ and ${ }^{237} \mathrm{Cm}$ have been unambiguously identified. ${ }^{4-6}$

In this report, the present status of nuclear chemistry studies of heavy elements and research plans for the near future at JAERI are summarized.

\section{Production of ${ }^{261} \mathrm{Rf}$ and ${ }^{262} \mathrm{Db}$}

As shown in Figure 1, the ${ }^{248} \mathrm{Cm}$ target of $590 \mu \mathrm{g} / \mathrm{cm}^{2}$ thickness prepared by electrodeposition onto a $2.2 \mathrm{mg} / \mathrm{cm}^{2}$ thick beryllium backing foil was bombarded by ${ }^{18} \mathrm{O}$ and ${ }^{19} \mathrm{~F}$ beams de-

*Corresponding author. E-mail: nagame@popsvr.tokai.jaeri.go.jp. FAX: +81-29-282-5927.

tPermanent address: Department of Chemistry, Niigata University, Niigata 950-2181, Japan.

†Permanent address: Department of Radiologic Science and Engineering, University of Tokushima, Tokushima 770-8509, Japan. livered from the JAERI tandem accelerator. The reaction products recoiling out of the target were stopped in He gas ( $\sim 1$ bar), attached to $\mathrm{KCl}$ aerosols, and were transported through a Teflon capillary to the rotating wheel apparatus MANON. The transported nuclei were deposited on polyethylene terephthalate foils of $120 \mu \mathrm{g} / \mathrm{cm}^{2}$ thickness. The wheel was periodically rotated to position the foils between six pairs of Si PIN photodiodes for $\alpha$-particle detection. The production cross sections were evaluated from the mother-daughter correlations of $\alpha$ energies between ${ }^{261} \mathrm{Rf}$ and ${ }^{257} \mathrm{No}$, and ${ }^{262} \mathrm{Db}$ and ${ }^{258} \mathrm{Lr}$. The experimental details are described in Reference 2.

The typical $\alpha$-particle spectrum measured in the six top detectors during irradiation of $1.6 \times 10^{17}{ }^{19} \mathrm{~F}$ ions for the production of ${ }^{262} \mathrm{Db}$ in the $106 \mathrm{MeV}{ }^{19} \mathrm{~F}$-induced reaction of ${ }^{248} \mathrm{Cm}$ is shown in Figure 2(a). In the $\alpha$-energy range of $8.40-8.70 \mathrm{MeV}, \alpha$ lines from $34-\mathrm{s}{ }^{262} \mathrm{Db}(8.45,8.53$, and $8.67 \mathrm{MeV})$ and its daughter $3.9-\mathrm{s}{ }^{258} \operatorname{Lr}(8.565,8.595,8.621$, and $8.654 \mathrm{MeV})$ are observed. Figure 2(b) shows the mother-daughter $(\alpha-\alpha)$ correlation plot for the $106 \mathrm{MeV}{ }^{19} \mathrm{~F}+{ }^{248} \mathrm{Cm}$ reaction. A total of $30 \alpha-\alpha$ correlation events were registered. The literature values for the $\alpha$ energies of ${ }^{262} \mathrm{Db}$ and ${ }^{258} \mathrm{Lr}$ are indicated by the solid lines.

From the mother-daughter correlation events, the production cross section of ${ }^{261} \mathrm{Rf}$ was evaluated to be $13 \pm 3 \mathrm{nb}$ at the ${ }^{18} \mathrm{O}$ energy of $94 \mathrm{MeV}$, while that of ${ }^{262} \mathrm{Db}$ was $1.3 \pm 0.4 \mathrm{nb}$ at $106 \mathrm{MeV}$ ${ }^{19} \mathrm{~F}$ (Ref. 2).

\section{Aqueous Chemistry of Rf}

Study of the chemical properties of the transactinide elements is challenging both experimentally and theoretically. ${ }^{7}$ Due to in-
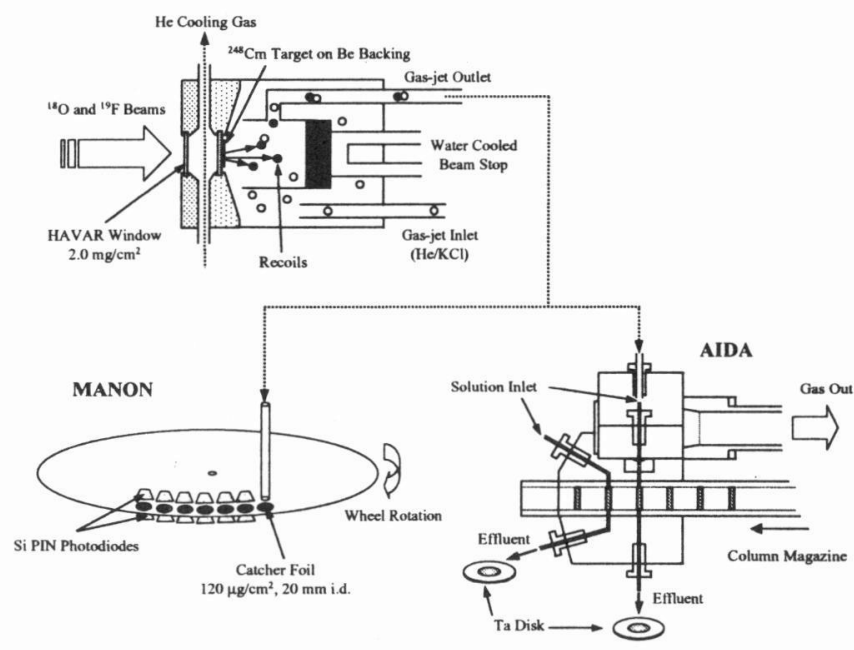

Figure 1. Schematic drawing of the experimental setup. 

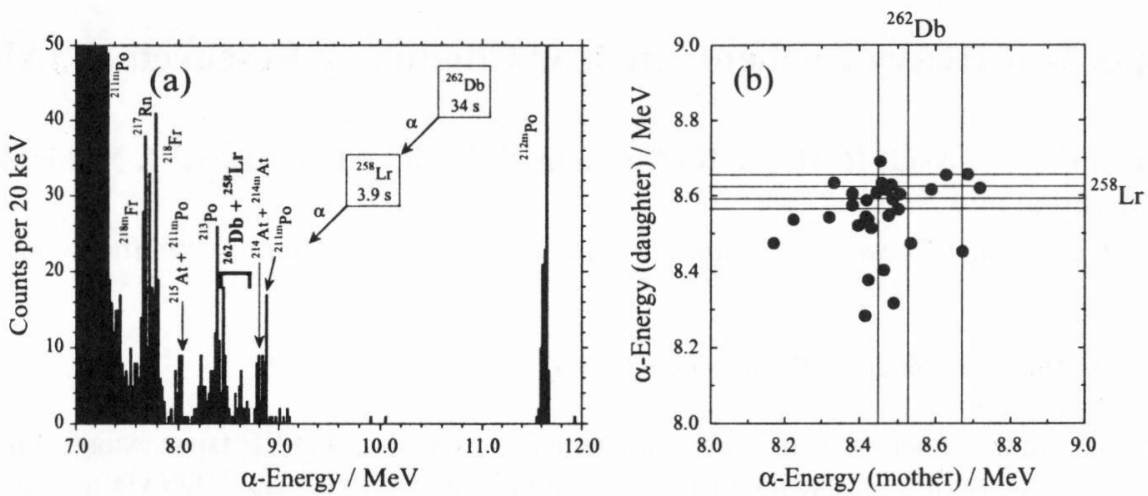

Figure 2. (a) Sum of $\alpha$-particle spectra measured in the bombardment of the ${ }^{248} \mathrm{Cm}$ target with $106 \mathrm{MeV}{ }^{19} \mathrm{~F}$ ions. (b) Mother-daughter correlations for the above reaction. The $\alpha$ energies of the mother and daughter for each correlated event are plotted. The literature values of the mother and daughter $\alpha$-particle energies are shown by the solid lines.

creasingly strong relativistic effects, increasing deviations from the periodicity of the chemical properties based on extrapolations from the lighter homologues are predicted. Thus, the experimental approach should involve a detailed comparison of the chemical properties of the transactinides with those of their lighter homologues under identical conditions. We have investigated the anion-exchange behavior of $\mathrm{Rf}$ together with the lighter homologues $\mathrm{Zr}$ and $\mathrm{Hf}$ in the same on-line experiments. ${ }^{3}$

To perform fast and repetitive ion-exchange separation of Rf, we have developed the apparatus AIDA that consists of a modified ARCA (Automated Rapid Chemistry Apparatus) which is the miniaturized computer-controlled liquid chromatography system $^{8}$ and an automated on-line $\alpha$-particle detection system.

In the following, we briefly introduce the experiment and a part of the result on the anion-exchange behavior of $\mathrm{Rf}$ in $\mathrm{HCl}$ solution. $78-\mathrm{s}{ }^{261} \mathrm{Rf}$ was produced in the $94 \mathrm{MeV}{ }^{18} \mathrm{O}$-induced reaction of ${ }^{248} \mathrm{Cm}$. As shown in Figure 1, the reaction products recoiling out of the target were transported by the $\mathrm{He} / \mathrm{KCl}$ gasjet system to the collection site of AIDA, where the products were dissolved with $170 \mu \mathrm{L}$ of hot $\left(\sim 80^{\circ} \mathrm{C}\right) 11.5 \mathrm{M} \mathrm{HCl}$ and were fed onto the $1.6 \mathrm{~mm} \phi \times 7 \mathrm{~mm}$ chromatographic column filled with the anion exchange resin MCl GEL CA08Y (particle
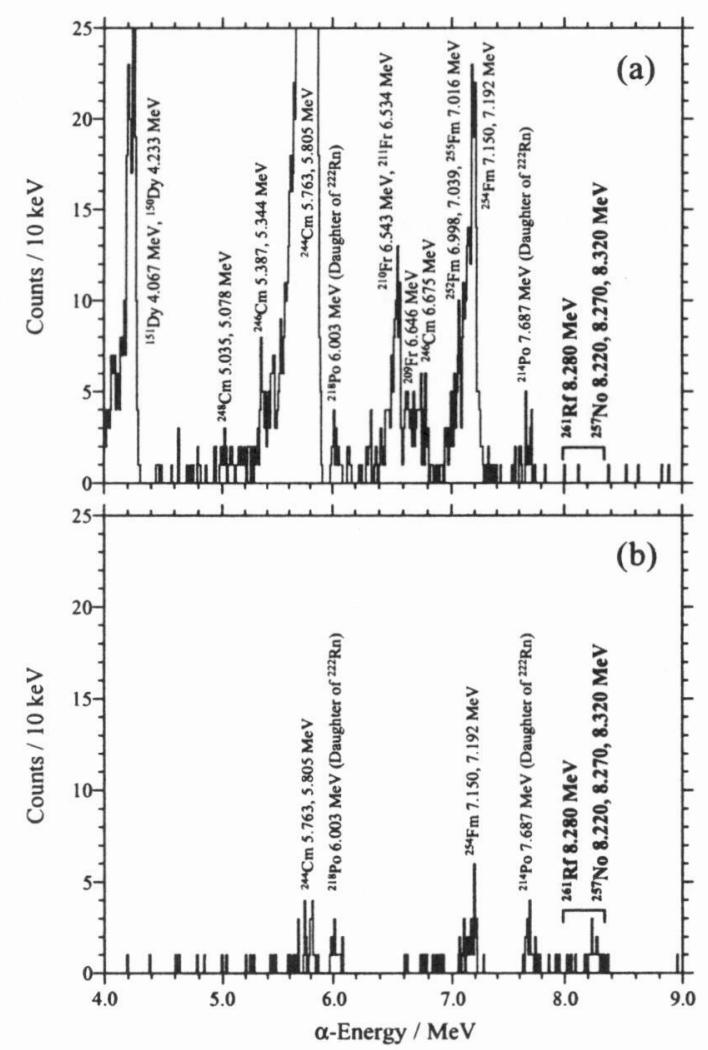

Figure 3. $\alpha$-particle spectra of samples prepared from the two elution fractions: (a) $11.5 \mathrm{M} \mathrm{HCl}$ and (b) $4.0 \mathrm{M} \mathrm{HCl}$. size: about $20 \mu \mathrm{m}$ ) at a flow rate of $1.0 \mathrm{~mL} / \mathrm{min}$. The effluent collected on a Ta disk as fraction 1 was evaporated to dryness, and the remaining products in the column were eluted with $280 \mu \mathrm{L}$ of $4.0 \mathrm{M} \mathrm{HCl}$ as fraction 2 . The pair of Ta disks were automatically subjected to $\alpha$ spectrometry with eight $600-\mathrm{mm}^{2}$ passivated ion-implanted planar silicon (PIPS) detectors.

Figures 3(a) and 3(b) show the $\alpha$ spectra observed in the first fraction with $11.5 \mathrm{M} \mathrm{HCl}$ and that in the second fraction with $4.0 \mathrm{M} \mathrm{HCl}$, respectively. There are only a few $\alpha$ events from ${ }^{261} \mathrm{Rf}-{ }^{257} \mathrm{No}$ at $11.5 \mathrm{M} \mathrm{HCl}$, which means $\mathrm{Rf}$ is strongly adsorbed by the anion-exchange resin, while at $4.0 \mathrm{M} \mathrm{HCl}, \mathrm{Rf}$ is not retained in the column. The contribution of the $\alpha$ events from ${ }^{257}$ No formed from the $\alpha$ decay of ${ }^{261} \mathrm{Rf}$ before the chemical processing was negligible. ${ }^{9}$ Further we confirmed that No was entirely eluted with $11.5 \mathrm{M} \mathrm{HCl}^{9}$ As shown in Figure 3(a), the $\mathrm{Cm}$ isotopes, recoil products of the target (the isotopic composition of the ${ }^{248} \mathrm{Cm}$ target is follows: ${ }^{244} \mathrm{Cm}$ (1.12 at.\%) and ${ }^{246} \mathrm{Cm}(1.31$ at. \%) ) and the $\mathrm{Fm}$ isotopes, transfer reaction products from the $\mathrm{Cm}$ target are mostly eluted with $11.5 \mathrm{M} \mathrm{HCl}$. The present result is consistent with the fact that the chloride complexation of element 104 in $12 \mathrm{M} \mathrm{HCl}$ was stronger than that of the trivalent actinides. ${ }^{10}$ The rare earth element isotopes ${ }^{150,151} \mathrm{Dy}$, the transfer reaction products from ${ }^{152} \mathrm{Gd}$ in the ${ }^{248} \mathrm{Cm}$ target are also eluted with $11.5 \mathrm{M} \mathrm{HCl}$. The on-line experiments with $\mathrm{Zf}$ and $\mathrm{Hf}$ isotopes were carried out under identical conditions as those with ${ }^{261} \operatorname{Rf}$ (Ref. 3).

As reported in Reference 3, the adsorption behavior of Rf as a function of $\mathrm{HCl}$ concentration was similar to that of the group 4 elements $\mathrm{Zr}$ and $\mathrm{Hf}$, and was quite different from that of the tetravalent pseudo-homologue $\mathrm{Th}$ in $\mathrm{HCl}$ concentration of $>8 \mathrm{M}$, indicating that the anionic chloride complexes of the tetravalent $\mathrm{Rf}, \mathrm{Zr}$, and $\mathrm{Hf}$ are formed in concentrated $\mathrm{HCl}$. The

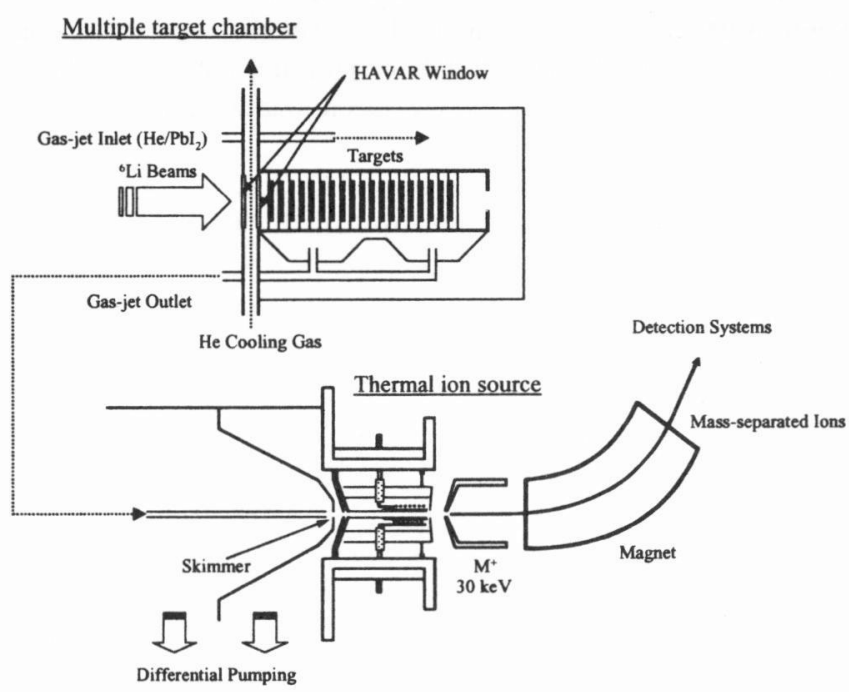

Figure 4. Schematic view of the gas-jet coupled JAERI-ISOL. 
TABLE 1: EC/ $\alpha$ decay properties of the neutron-deficient Am and Cm isotopes measured with JAERI-ISOL and those compared with the literature data.

\begin{tabular}{|c|c|c|c|c|c|c|}
\hline \multirow[t]{2}{*}{ Nuclide } & \multicolumn{2}{|c|}{ Half-life / min } & \multicolumn{2}{|c|}{$\alpha$-energy / MeV } & \multicolumn{2}{|c|}{$\alpha$-branching ratio / \% } \\
\hline & JAERI-ISOL & Reference & JAERI-ISOL & Reference & JAERI-ISOL & Reference \\
\hline${ }^{233} \mathrm{Am}$ & $3.2 \pm 0.8^{6}$ & $(1.72)^{a}$ & $6.780 \pm 0.017^{6}$ & $(7.100)^{b}$ & $>3^{6}$ & \\
\hline${ }^{234} \mathrm{Am}$ & & $2.32 \pm 0.08^{18}$ & & $6.460^{18}$ & $<0.04^{16}$ & $0.039 \pm 0.012^{18}$ \\
\hline${ }^{235} \mathrm{Am}$ & $10.3 \pm 0.6^{16}$ & $15 \pm 5^{22}$ & $6.457 \pm 0.014^{16}$ & $(6.700)^{b}$ & $0.40 \pm 0.05^{16}$ & \\
\hline${ }^{236 g} \mathrm{Am}$ & $3.6 \pm 0.2^{17}$ & $4.4 \pm 0.8^{5}$ & $6.150^{16}$ & $6.41^{19}$ & $0.004^{16}$ & $0.042 \pm 0.006^{19}$ \\
\hline${ }^{236 m} \mathrm{Am}$ & $2.9 \pm 0.2^{17}$ & & & & & \\
\hline${ }^{237} \mathrm{Cm}$ & & $(15)^{a}$ & $6.660 \pm 0.010^{4}$ & $(6.800)^{b}$ & & \\
\hline${ }^{238} \mathrm{Cm}$ & & & $6.560 \pm 0.010^{4}$ & $6.520 \pm 0.050^{23}$ & & \\
\hline
\end{tabular}

${ }^{a}$ Predicted. ${ }^{20}$

${ }^{b}$ Evaluated from the $Q$ values in Reference 21 .

other interesting feature was observed in the adsorption trend on the anionic-exchange resin among $\mathrm{Rf}, \mathrm{Zr}$, and $\mathrm{Hf}$. The adsorption order that reflects the stability of chloride complexation was $\mathrm{Rf}>\mathrm{Zr}>\mathrm{Hf}$. Presently, we are conducting the relativistic molecular orbital calculations to understand the present results and to see if the anion-exchange behavior of Rf is affected by relativistic effects.

\section{EC/ $\alpha$-decay Studies of Neutron-deficient Actinides}

There still remain many unknown isotopes to be discovered in the region of neutron-deficient actinides which predominantly decay through the electron capture (EC). Decay properties of these nuclides lead to considerable advances in the understanding of proton excess heavy nuclei: verification of the proton drip line, nuclear structure of large deformed nuclei such as hexadecapole deformation, and fission barrier heights of neutrondeficient nuclei far from stability.

To search for new isotopes and study EC/ $\alpha$-decay properties of neutron-deficient actinides, we have developed a composite system consisting of a gas-jet transport apparatus and a thermal ion source in the on-line isotope separator (JAERI-ISOL). ${ }^{5,11}$ This gas-jet coupled JAERI-ISOL system enables us to determine simultaneously mass number via the isotope separator and atomic number by the measurement of $\mathrm{x}$ rays associated with the $E C / \beta^{ \pm}$decay of a nucleus. Some new neutron-rich rareearth isotopes produced in the proton-induced fission of ${ }^{238} \mathrm{U}$ were identified with this system. ${ }^{12-15}$ In the following, the EC/ $\alpha$ decay studies of the neutron-deficient actinides are summarized.

The experimental setup is schematically drawn in Figure 4. The ${ }^{6} \mathrm{Li}$ beams delivered from the JAERI tandem accelerator bombarded the ${ }^{233,235} \mathrm{U}$ and ${ }^{237} \mathrm{~Np}$ targets set in a multiple target chamber. The used reaction systems are ${ }^{233} \mathrm{U}\left({ }^{6} \mathrm{Li}, x n\right){ }^{233-235} \mathrm{Am}$,

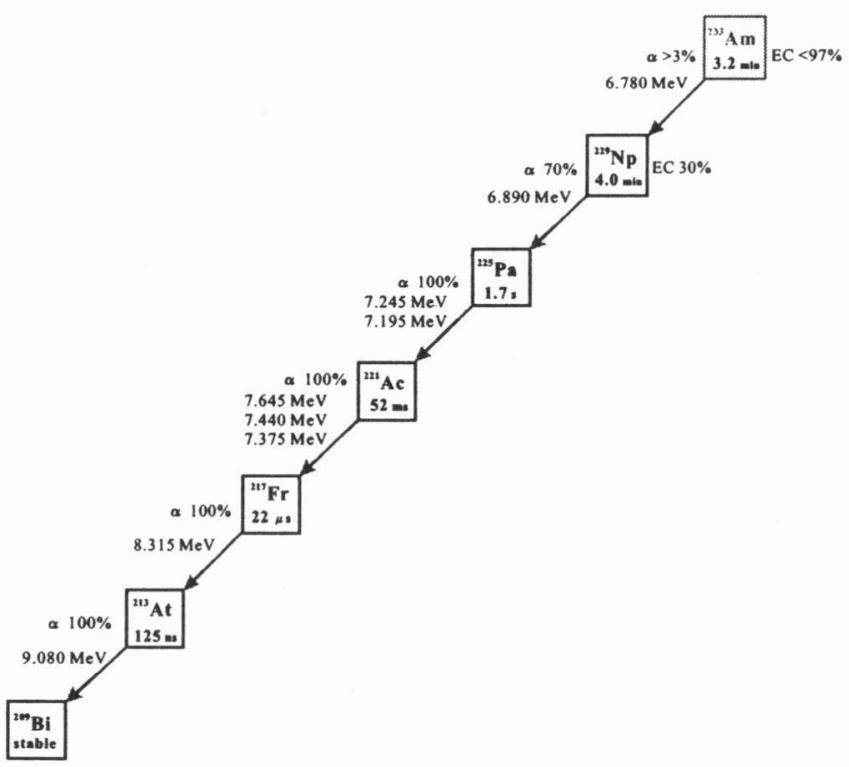

Figure 5. $\alpha$-decay chain originating from the $\alpha$ decay of ${ }^{233} \mathrm{Am}$.
${ }^{235} \mathrm{U}\left({ }^{6} \mathrm{Li}, 5 n\right){ }^{236} \mathrm{Am}$, and ${ }^{237} \mathrm{~Np}\left({ }^{6} \mathrm{Li}, x n\right){ }^{237,238} \mathrm{Cm}$. The reaction products recoiling out of the targets were thermalized in He gas loaded with $\mathrm{PbI}_{2}$ aerosol clusters. The products attached to the aerosols were swept out of the target chamber and transported to the thermal ion source of ISOL through a Teflon capillary ( $1.5 \mathrm{~mm}$ i.d. and $8 \mathrm{~m}$ length). The transported nuclides were ionized in the thermal ion source, and mass-separated atoms were collected on an aluminum coated Mylar tape in a tape transport system or a rotating catcher foil apparatus. In the tape transport system, we used Ge detectors for the $\mathrm{x} / \gamma$-ray measurement and Si PIN photodiode detectors set in the rotating catcher foil apparatus were employed for the measurement of $\alpha$ particles. Detailed experimental procedures are described in References 6,11.

As a typical example, the identification of the new isotope ${ }^{233} \mathrm{Am}$ produced in the ${ }^{233} \mathrm{U}\left({ }^{6} \mathrm{Li}, 6 n\right)$ reaction is given in the follows. The isotope ${ }^{233} \mathrm{Am}$ was identified through an $\alpha-\alpha$ correlation analysis. The $\alpha$ decay of ${ }^{233} \mathrm{Am}$ is followed by the five successive $\alpha$ decays starting from ${ }^{229} \mathrm{~Np}$ as shown in Figure $5:{ }^{229} \mathrm{~Np}$ $\left(T_{1 / 2}=4.0 \mathrm{~min}\right) \rightarrow{ }^{225} \mathrm{~Pa}\left(T_{1 / 2}=1.7 \mathrm{~s}\right) \rightarrow{ }^{221} \mathrm{Ac}\left(T_{1 / 2}=52 \mathrm{~ms}\right)$ $\rightarrow{ }^{217} \mathrm{Fr}\left(T_{1 / 2}=22 \mu \mathrm{s}\right) \rightarrow{ }^{213} \mathrm{At}\left(T_{1 / 2}=125 \mathrm{~ns}\right) \rightarrow{ }^{209} \mathrm{Bi}$ (stable). Since the last four nuclides decay via $\alpha$-particle emission with the short half-lives, the $\alpha-\alpha$ correlation events among these nuclides can be unambiguously identified. Figure 6 shows an $\alpha$ particle spectrum constructed from the observed $\alpha-\alpha$ correlation events in the mass-separated $A=233$ fraction. The $\alpha$-particle energies of ${ }^{233} \mathrm{Am}$ and ${ }^{229} \mathrm{~Np}$ are clearly observed and those corresponding to the decays of the other nuclides are also seen. The $\alpha$ energy of ${ }^{233} \mathrm{Am}$ was determined to be $6.780 \pm 0.017 \mathrm{MeV}$, and from the decay curve of the intensity of this $\alpha$ line, the halflife was $3.2 \pm 0.8 \mathrm{~min}$. Since no Pu Kx rays following the EC decay of ${ }^{233} \mathrm{Am}$ was observed, the $\alpha$-decay branching ratio was estimated as $I_{\alpha}>3 \%$ based on the detection efficiency of the Pu Kx rays. ${ }^{6}$

In Table 1 , the half-lives, $\alpha$-decay energies, and $\alpha$-decay branching ratios measured with JAERI-ISOL and those from the literature data are summarized. In the case of ${ }^{235} \mathrm{Am}$, the $\alpha$-decay process was observed for the first time and the $\alpha$ -

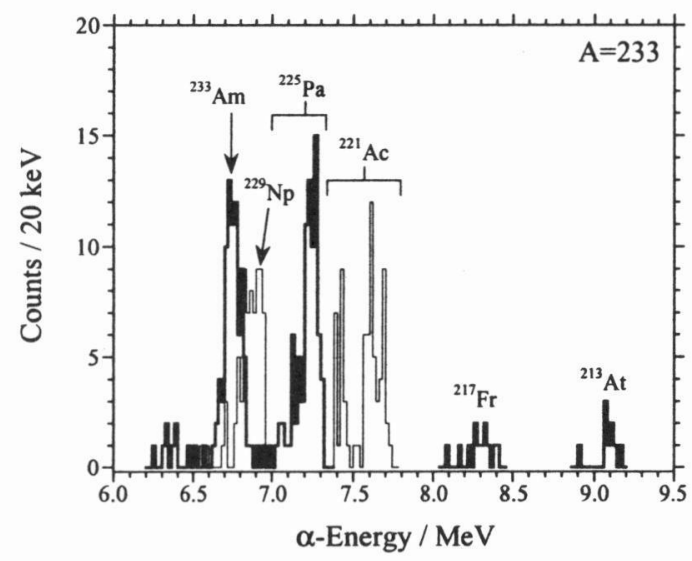

Figure 6. $\alpha$-particle spectrum constructed from the measured $\alpha-\alpha$ correlation events associated with the $\alpha$ decay of ${ }^{233} \mathrm{Am}$. 
branching intensity was derived from the ratio between the observed $\alpha$ and Pu Kx-ray intensities. ${ }^{16}$ The half-life value of ${ }^{235} \mathrm{Am}$ was determined to be $10.3 \pm 0.6 \mathrm{~min}$ based on the decay curve of the $\alpha$ line of $6.457 \mathrm{MeV}$ and that of $\mathrm{Pu} \mathrm{K}_{\alpha} \mathrm{x}$ rays following the EC decay of ${ }^{235} \mathrm{Am}$. With the $\gamma-\gamma$ coincidence technique, it was found that there are two EC-decaying states in ${ }^{236} \mathrm{Am}$ (Ref. 17). The $\alpha$-decay process in ${ }^{234} \mathrm{Am}$ was not observed, although that was reported by Hall et al. ${ }^{18}$ as shown in Table 1 . In the $\alpha$ decay of ${ }^{236} \mathrm{Am}$, a very weak $\alpha$ group was observed at $6.150 \mathrm{MeV}$, while the $6.41 \mathrm{MeV} \alpha$ group was measured in Reference 19.

\section{Future Plans}

In the study of Rf aqueous chemistry, anion-exchange experiments in HF solution will be soon conducted. Plans for additional experiments are being made to understand complex structure of $\mathrm{Rf}$ together with $\mathrm{Zr}$ and $\mathrm{Hf}$ in $\mathrm{HNO}_{3}, \mathrm{HCl}$, and $\mathrm{HF}$ solutions. Measurements of XAFS (X-ray Absorption Fine Structure) spectra of $\mathrm{Zr}$ and $\mathrm{Hf}$ compounds in these solutions will be performed at KEK (High Energy Accelerator Research Organization) Photon Factory. Relativistic molecular orbital calculations are also being carried out to gain understanding of complex chemistry of Rf, and to see if there are unexpected differences within the group 4 elements.

In the next stage of aqueous chemistry, we plan to conduct $\mathrm{Db}$ ion-exchange experiments. Improvements to the overall production rates and a possible multiple target system are being considered to study the short-lived $34-\mathrm{s}{ }^{262} \mathrm{Db}$ produced in the ${ }^{248} \mathrm{Cm}\left({ }^{19} \mathrm{~F}, 5 n\right)$ reaction with the small cross section of about $1.5 \mathrm{nb}$. Development of a new ion-exchange separation apparatus, an improved AIDA system is required. We are now cooperating with the Niigata group on the gas phase chemistry of the transactinide elements. An apparatus based on the isothermal gas chromatography is now under construction.

Experiments of $\alpha-\gamma$ spectroscopy, and spontaneous fission and $\alpha$-decay properties of heavy and transactinide nuclei will be conducted with the gas-jet coupled JAERI-ISOL, an $\alpha-\gamma$ spectrometry apparatus, and MANON coupled with a gas chemistry separator system.

Acknowledgement. The present study has been carried out in collaboration with Niigata University, Osaka University, Tokyo Metropolitan University, University of Tokushima, Kanazawa University, Nagoya University, Hiroshima University, Gesellschaft für Schwerionenforschung (GSI), Mainz University, Paul Scherrer Institut (PSI), Bern University, and Institute of High Energy Physics, Beijing. We would like to acknowledge the crew of the JAERI tandem accelerator for providing the stable and intense ${ }^{6} \mathrm{Li},{ }^{18} \mathrm{O}$, and ${ }^{19} \mathrm{~F}$ beams. This work was partly supported by the JAERI tandem accelerator collaboration program and the Program on the Scientific Cooperation between JAERI and GSI in Research and Development in the Field of Ion Beam Application.

\section{References}

(1) H. Haba, K. Tsukada, M. Asai, I. Nishinaka, M. Sakama S. Goto, M. Hirata, S. Ichikawa, Y. Nagame, T. Kaneko, H. Kudo, A. Toyoshima, Y. Shoji, A. Yokoyama, A. Shinohara, Y. Oura, K. Sueki, H. Nakahara, M. Schädel, J. V. Kratz, A. Türler, and H. W. Gäggeler, Radiochim. Acta 89, 733 (2001).

(2) Y. Nagame, M. Asai, H. Haba, S. Goto, K. Tsukada, I. Nishinaka, K. Nishio, S. Ichikawa, A. Toyoshima, K. Akiyama, H. Nakahara, M. Sakama, M. Schädel, J. V. Kratz, H. W. Gäggeler, and A. Türler, J. Nucl. Radiochem. Sci. 3, 85 (2002).

(3) H. Haba, K. Tsukada, M. Asai, S. Goto, A. Toyoshima, I. Nishinaka, K. Akiyama, M. Hirata, S. Ichikawa, Y.
Nagame, Y. Shoji, M. Shigekawa, T. Koike, M. Iwasaki, A. Shinohara, T. Kaneko, T. Maruyama, S. Ono, H. Kudo, Y. Oura, K. Sueki, H. Nakahara, M. Sakama, A. Yokoyama, J. V. Kratz, M. Schädel, and W. Brüchle, J. Nucl. Radiochem. Sci. 3, 143 (2002).

(4) M. Asai, M. Sakama, K. Tsukada, S. Ichikawa, H. Haba, I. Nishinaka, Y. Nagame, S. Goto, K. Akiyama, A. Toyoshima, Y. Kojima, Y. Oura, H. Nakahara, M. Shibata, and K. Kawade, J. Nucl. Radiochem. Sci. 3, 187 (2002).

(5) K. Tsukada, S. Ichikawa, Y. Hatsukawa, I. Nishinaka, K. Hata, Y. Nagame, Y. Oura, T. Ohyama, K. Sueki, H. Nakahara, M. Asai, Y. Kojima, T. Hirose, H. Yamamoto, and K. Kawade, Phys. Rev. C 57, 2057 (1998).

(6) M. Sakama, K. Tsukada, M. Asai, S. Ichikawa, H. Haba, S. Goto, Y. Oura, I. Nishinaka, Y. Nagame, M. Shibata, Y. Kojima, K. Kawade, M. Ebihara, and H. Nakahara, Eur. Phys. J. A 9, 303 (2000).

(7) J. V. Kratz, Heavy Elements and Related New Phenomena, edited by W. Greiner and R. K. Gupta (World Scientific, Singapore, 1999), p. 129.

(8) M. Schädel, W. Brüchle, E. Jäger, E. Shimpf, J. V. Krtaz, U. W. Scherer, and H. P. Zimmermann, Radiochim. Acta 48, 171 (1989).

(9) A. Toyoshima, K. Tsukada, H. Haba, M. Asai, S. Goto, K. Akiyama, I. Nishinaka, S. Ichikawa, Y. Nagame, and A. Shinohara, J. Radioanal. Nucl. Chem. (in press).

(10) E. K. Hulet, R. W. Lougheed, J. F. Wild, J. H. Landrum, J. M. Nitschke, and A. Ghiorso, J. Inorg. Nucl. Chem. 42, 79 (1980).

(11) S. Ichikawa, K. Tsukada, M. Asai, H. Haba, M. Sakama, Y. Kojima, M. Shibata, Y. Nagame, Y. Oura, and K. Kawade, Nucl. Instrum. Methods B 187, 548 (2002).

(12) M. Asai, K. Tsukada, S. Ichikawa, A. Osa, Y. Kojima, M. Shibata, H. Yamamoto, K. Kawade, N. Shinohara, Y. Nagame, H. Iimura, Y. Hatsukawa, and I. Nishinaka, J. Phys. Soc. Jpn. 65, 1135 (1996).

(13) S. Ichikawa, K. Tsukada, I. Nishinaka, Y. Hatsukawa, H. Iimura, K. Hata, Y. Nagame, A. Osa, M. Asai, Y. Kojima, T. Hirose, M. Shibata, K. Kawade, and Y. Oura, Phys. Rev. C 58, 1329 (1998).

(14) M. Asai, S. Ichikawa, K. Tsukada, M. Sakama, M. Shibata, Y. Kojima, A. Osa, I. Nishinaka, Y. Nagame, K. Kawade, and T. Tachibana, Phys. Rev. C 59, 3060 (1999).

(15) M. Asai, K. Tsukada, S. Ichikawa, H. Haba, A. Osa, Y. Nagame, S. Goto, M. Sakama, Y. Kojima, M. Shibata, K. Akiyama, and A. Toyoshima, JAERI-Review 2001-030, 13 (2001).

(16) M. Sakama, M. Asai, K. Tsukada, S. Ichikawa, H. Haba, S. Goto, I. Nishinaka, Y. Nagame, Y. Oura, M. Ebihara, H. Nakahara, M. Shibata, K. Kawade, and Y. Kojima (to be submitted).

(17) M. Asai, M. Sakama, K. Tsukada, S. Ichikawa, H. Haba, I. Nishinaka, Y. Nagame, S. Goto, Y. Kojima, Y. Oura, H. Nakahara, M. Shibata, and K. Kawade (submitted to Phys. Rev. C).

(18) H. L. Hall, K. E. Gregorich, R. A. Henderson, C. M. Gannett, R. B. Chadwick, J. D. Leyba, K. R. Czerwinski, B. Kadkhodayan, S. A. Kreek, D. M. Lee, M. J. Nurmia, D. C. Hoffman, C. E. A. Palmer, and P. A. Baisden, Phys. Rev. C 41, 618 (1990).

(19) H. L. Hall, LBL-27878 (1989).

(20) T. Tachibana, M. Yamada, and Y. Yoshida, Prog. Theor. Phys. 84, 641 (1990).

(21) G. Audi, O. Bersillon, J. Blachot, and A. H. Wapstra, Nucl. Phys. A 624, 1 (1997).

(22) J. Guo, Z. Gan, H. Liu, W. Yang, L. Shi, W. Mu, T. Guo, K. Fang, S. Shen, S. Yuan, X. Zhang, Z. Qin, R. Ma, J. Zhong, S. Wang, D. Kong, and J. Qiao, Z. Phys. A 355, 111 (1996).

(23) G. H. Higgins, UCRL 1796 (1952). 